\title{
Robust Dense Depth Acquisition Using 2-D De Bruijn Structured Light
}

\author{
Zhiliang Xu, Lizhuang Ma, and Wuzheng Tan \\ Department of Computer Science \& Engineering \\ Shanghai Jiaotong University \\ No. 800, Dongchuan Rd., Shanghai 200240, P.R. China \\ \{xuzhiliang, ma-lz, tanwuzheng\}@sjtu.edu.cn
}

\begin{abstract}
We present a new dense depth acquisition method using 2-D De Bruijn structured light, which is robust to various textures and is able to reconstruct dense depth maps of moving and deforming objects. A 2-D binary De Bruijn pattern is emitted to the target object by an off-the-shelf projector. Fast dynamic programming based stereo matching is performed on images taken from two different views. The depth is obtained by robust least square triangulation. The advantages include that we do not need to take image sequences with different illumination patterns and do not assume that the surface for reconstruction has uniform texture. Experimental results show that shapes can be efficiently obtained in good quality by the proposed approach. We believe that our approach is a good choice in applications of acquiring depth maps for moving scenes with inexpensive equipments.
\end{abstract}

Keywords: Depth acquisition, range sensing, 3-D model reconstruction, De Bruijn sequence.

\section{Introduction}

\subsection{Related Work}

Scene depth acquisition is a rapidly expanding field, with applications in robotics, modeling and virtual reality. Among them, binocular stereo [1, 2] is a convenient and inexpensive approach. It is also a hot field of recent researches.

Traditional passive stereo suffers from ambiguities in large textureless areas. To solve this problem, structured light is projected onto the object to endow it with a coded pattern, which is referred to as active stereo 3]. To obtain dense depth maps, time multiplex schemes are commonly used, such as Rusinkiewicz et al. 4] and Scharstein and Szeliski [5. In their works, since a series of patterns are projected sequentially, the objects to be reconstructed is either still or restricted to only slow motion, compared to the patterns cycled in $60 \mathrm{~Hz}$.

Generally, in active stereo, it is assumed that the surfaces to be reconstructed have uniform texture. Otherwise, the pattern received by the camera is hard to decode. To deal with the texture problem in active stereo scheme, Scharstein and Szeliski 5] project a series of black and white stripes as well as their inverses, 
which requires about 20 patterns to be projected sequentially to obtain the dense depth map with $1024 \times 768$ resolution.

Other related works include Lavoie et al. 6] and Pagès et al. [7. Their methods are similar to ours in that they use only one light pattern and take a single image of the illuminated object. However, Lavoie et al. 6] encode the structured light on the intersections of a grid pattern, and match the code only where reliable decoding is available. Thus a sparse reconstruction is obtained as the result. Pagès et al. [7] use 1-D color De Bruijn sequences so that the length of the pattern can cover the whole image and more information can be retrieved, and thus give a dense reconstruction. But color patterns are very sensitive to the textures on the surface.

\subsection{Overview of Our Approach}

In this paper, we propose a dense depth acquisition method which needs only a single image pair taken simultaneously, and avoids the difficulty in decoding the structured light pattern. As a result, our method is able to acquire depth maps of moving and deforming objects.

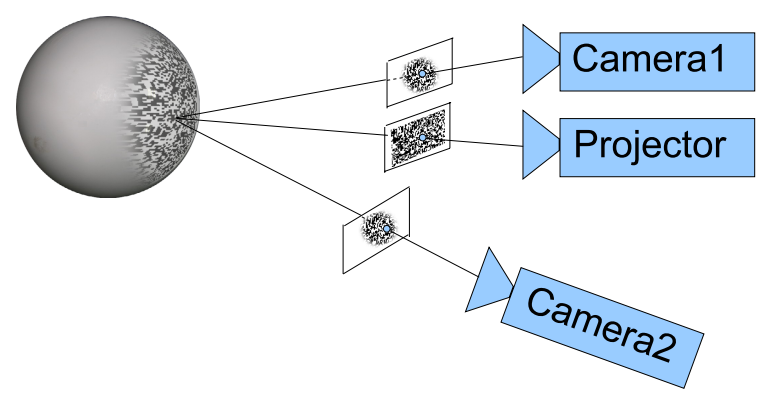

Fig. 1. Equipment setup of the dense depth acquisition system. The projector emits a 2-D binary De Bruijn pattern to the target object (a sphere). Camera \#1 (optional) is placed closely above the projector. Camera \#2 is located at an angle (about $20^{\circ}$ ) of the projector.

For hardware, we only require one off-the-shelf projector and at least one offthe-shelf camera. Another camera is needed optionally if the user is unwilling to calibrate the projector. The experiment setup is shown in Fig. 11 The projector emits a 2-D De Bruijn pattern onto the target object. One camera (the optional one) is located above the projector, as close as possible. Another camera is placed at an angle (typically $20^{\circ}$ ) with respect of the first camera. From now on, we assume that two cameras are used. The alternative system using only one camera is only a simple modification, which will be discussed in section 3.2. In our experiments, these two cameras are synchronized. Stereo matching and triangulation is then performed on the two images taken simultaneously to acquire the depth of the object. 


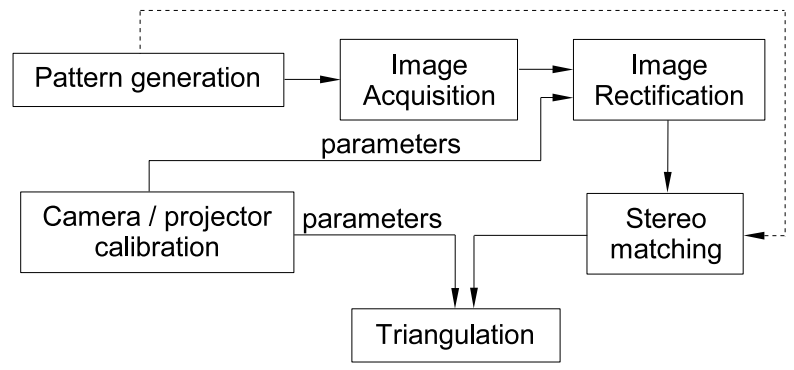

Fig. 2. The flow chart of our system. Two images are needed for stereo matching. The dotted line means that the projected pattern can be substituted by the image taken by Camera \#1.

The flow chart of our system is shown in Fig. 2. We first generate a binary 2-D De Bruijn pattern and emit it onto the target object. In the image acquisition step, two images of the target object are taken. These images are then rectified so that camera's radial distortion effect is removed and epipolar lines correspond to horizontal scanlines of images. Stereo matching based on normalized cross correlation (NCC) and dynamic programming (DP) is then performed on the stereo image pair. Finally, according to the disparities between the image pair and the recovered geometric parameters of the cameras and the projector, we use least square triangulation to reconstruct the metric depth values for every pixel of the object.

In the following, we will first introduce our 2-D binary De Bruijn pattern in section 2. Geometry calibration of cameras and projector is discussed in section 3 . We will introduce our NCC and DP based dense stereo matching algorithm in section 4 , and least square triangulation in section 5 . In section 6 , some experimental results are given to show the effectiveness of our approach. Finally, the paper is ended with a conclusion and discussion in section 7 .

Notations. In this paper, we refer to the image taken by the first camera, which is placed just above the projector as $I_{1}$, the image taken by another camera as $I_{2}$, and the image emitted by the projector $I_{p}$. The projection matrices of the cameras are denoted as $\mathbf{M}_{1}$ and $\mathbf{M}_{2}$ respectively.

\section{$2 \quad 2-D$ De Bruijn Sequences}

To reduce the ambiguities in stereo correspondence to the greatest extent, we need an illumination pattern with the property that every small local window appears only once in the whole pattern. Moreover, the pattern should be binarycolored so that it is robust to various surface textures. Traditional structured light patterns based on 1-D binary De Bruijn sequences are not enough, since they are limited on the length. In theory, the length of a 1-D De Bruijn sequence with unique sub-window size of 5 is only $32\left(=2^{5}\right)$, which is too short to cover 


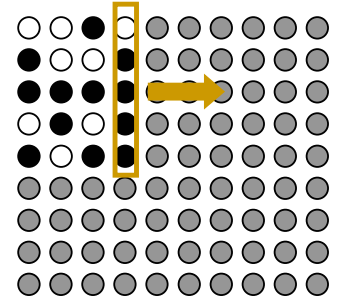

(a) Fill each column for the first 5 lines

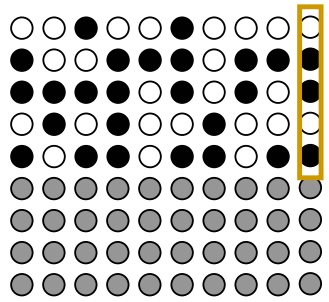

(b) Finish filling first 5 lines

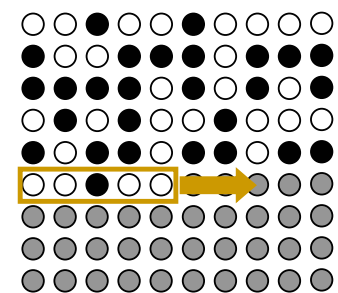

(c) Fill 5 bits each time for remaining lines

Fig. 3. Our algorithm for generating 2-D De Bruijn sequences

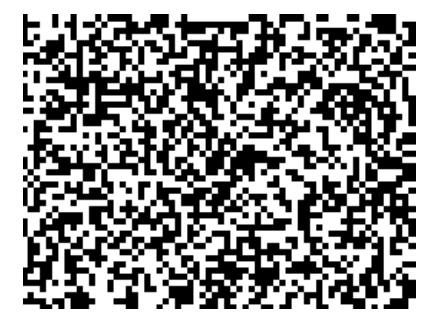

Fig. 4. A part of 2 -D binary De Bruijn sequence with $64 \times 48$ resolution. The pattern is enlarged to make every code bit distinguishable to the reader. Note that there is no repeated $5 \times 5$ sub-window in this pattern.

the whole image. However, if we use 2 -D De Bruijn sequence with $5 \times 5$ ( 5 rows by 5 columns) unique windows, the size becomes $2^{5 \times 5}(\approx 5792 \times 5792)$, which is adequately large for our application.

Although theoretically there exist 2-D De Bruijn sequences of $5 \times 5$ unique sub-windows with a large size, it is rather difficult to search for a valid one in practice. Here, we use a new algorithm for searching valid 2-D De Bruijn sequences, which is a modification of Morano et al. 88. Suppose that the size of unique sub-windows is $5 \times 5$, as illustrated in Fig. 3, the algorithm starts with randomly assigning the first 5 bits of the first column. We choose to fill 5 bits each time to avoid early termination and save the memory for back-tracking. For filling a new 5-bit group, we randomly generate a 5-bit code (we call it test bits, in contrast to those currently determined bits), and check whether $5 \times 5$ windows containing the test bits collide with all currently determined bits. If not, the 5 test bits are reserved and become determined bits, and then we go on to fill another 5-bit group. Otherwise, we generate another random 5-bit code, and test for collision again. If all the 32 possible 5 -bit codes have been tested, the algorithm comes to a failure. Then another trial should be aroused from scratch. After the first 5 rows are filled, each following rows are filled 5 -bit by 5 -bit in the same manner. Generally, about 5 to 8 trials are needed before obtaining such a part of valid 2-D De Bruijn sequence of size $512 \times 384$. A valid sequence can be obtained in about 5 minutes, which is satisfactory. A typical part of the pattern is shown in Fig. 4 . 


\section{Geometric Calibration}

In order to obtain the metric reconstruction of the object's depth, both intrinsic and extrinsic parameters of cameras and projector (optionally) are needed. Intrinsic parameters include focal length $f$, principal point $p_{0}=\left(u_{0}, v_{0}\right)$, skew coefficient $\alpha$ and distortions $\mathbf{k}$. In practice, we only consider the first order distortion $k_{1}$. Extrinsic parameters include the rotation matrix

$$
\mathbf{R}=\left(\begin{array}{lll}
r_{11} & r_{12} & r_{13} \\
r_{21} & r_{22} & r_{23} \\
r_{31} & r_{32} & r_{33}
\end{array}\right)
$$

and translation vector $T=\left(T_{x}, T_{y}, T_{z}\right)^{\prime}$ of camera with respect to the world coordinate, which is defined by a reference object, such as a checkerboard.

\subsection{Camera Calibration}

Camera geometric calibration has been well studied by Tsai [9, Zhang et al. 10, and Heikkilä and Silvén [11, etc. In this paper, we employ the four-step approach of Heikkilä and Silvén [11, which is more convenient. Actually, other calibration methods and even self-calibration [12] can also be adopted. The only additional equipment needed for camera calibration is a checkerboard. Omitting the skew effect, the relationship between world coordinate $P_{w}=\left(X_{w}, Y_{w}, Z_{w}, 1\right)^{\prime}$ and the pixel coordinate of its projection on the image $p=(u, v, 1)^{\prime}$ can be represented by

$$
\begin{aligned}
\left(1+k_{1} r^{2}\right) u & =f \frac{r_{11} X_{w}+r_{12} Y_{w}+r_{13} Z_{w}+T_{x}}{r_{31} X_{w}+r_{32} Y_{w}+r_{33} Z_{w}+T_{z}}, \\
\left(1+k_{1} r^{2}\right) v & =f \frac{r_{21} X_{w}+r_{22} Y_{w}+r_{23} Z_{w}+T_{y}}{r_{31} X_{w}+r_{32} Y_{w}+r_{33} Z_{w}+T_{z}} .
\end{aligned}
$$

where $r^{2}=u^{2}+v^{2}$. After we have estimated all the 14 unknowns using the method of [11, we rectify the radial distortion of the camera according to $k_{1}$, after which the camera model becomes a linear projection one, which can be written in matrix form as

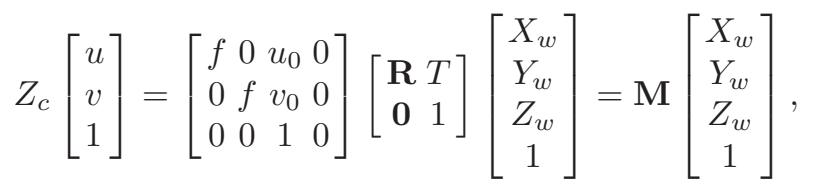

where

$$
Z_{c}=r_{31} X_{w}+r_{32} Y_{w}+r_{33} Z_{w}+T_{z},
$$

and

$$
\mathbf{M} \triangleq\left[\begin{array}{llll}
m_{11} & m_{12} & m_{13} & m_{14} \\
m_{21} & m_{22} & m_{23} & m_{24} \\
m_{31} & m_{32} & m_{33} & m_{34}
\end{array}\right]=\left[\begin{array}{llll}
f & 0 & u_{0} & 0 \\
0 & f & v_{0} & 0 \\
0 & 0 & 1 & 0
\end{array}\right]\left[\begin{array}{ll}
\mathbf{R} & T \\
\mathbf{0} & 1
\end{array}\right] .
$$

This rectification is crucial, since the computational cost in stereo matching and triangulation can be dramatically reduced if we use linear projection models for camera and projector. 


\subsection{Projector Calibration}

If only one camera is used in the system, as in many active stereo systems, stereo matching is performed between the image taken by camera and the pattern image emitted by the projector. That is, the role of image $I_{1}$ is replaced by $I_{p}$. In such a case, the projector has to be geometrically calibrated. Projector calibration is a tough task since the projector cannot take images of the scene. We solve this problem taking the advantage of our calibrated camera.

The projector is considered as the "reversed" camera, which projects the image on "CCD plane" back to the scene. We can use the same projection model for projector calibration. But unlike the camera, the world coordinates of the points projected into the scene have to be obtained with the help of the calibrated camera. We make the projector emit a checkerboard pattern onto the $Z_{w}=0$ plane. The other two coordinate components $\left(X_{w}\right.$ and $\left.Y_{w}\right)$ of the projected points on the plane are computed according to the pixel coordinates in the image taken by the calibrated camera. Formally, we solve for $X_{w}$ and $Y_{w}$ in the following linear equation set:

$$
Z_{c}\left[\begin{array}{l}
u \\
v \\
1
\end{array}\right]=\mathbf{M}_{2}\left[\begin{array}{c}
X_{w} \\
Y_{w} \\
0 \\
1
\end{array}\right],
$$

where $u$ and $v$ are the homogeneous pixel coordinates of the point's projection on the image taken by camera, and $\mathbf{M}_{2}$ is the projection matrix of the calibrated camera. $u, v$ and $\mathbf{M}_{2}$ are already known to us by corner extraction and camera calibration. Now that we have the world coordinates of the points corresponding to the projected pattern, projector calibration can be continued in the same manner as camera calibration. Here we assume that the projector has no radial distortion and skew effect.

The advantages of using one calibrated camera and one calibrated projector in stereo matching include saving one camera, and avoiding the efforts to synchronize two cameras. But the disadvantages are that the projector calibration is more complicated, and there exists no self-calibration technique for recovering the geometry parameters of the projector. Additionally, we need to ensure that the scaled images $I_{2}$ and $I_{p}$ have similar pattern sizes. Otherwise, the stereo matching algorithm will be confused.

\section{Stereo Matching}

Although the original structured light pattern has the unique sub-window property, new ambiguities are aroused after camera's re-sampling and distortion caused by different view angles. So we cannot directly decode the images. On the contrary, NCC based robust stereo matching is used instead.

For sake of efficiency, the two stereo images $I_{1}$ and $I_{2}$ are further rectified using the method of Loop and Zhang [10, so that each epipolar line corresponding to horizontal scanlines of the images, and the disparities between two views only resides in the horizontal direction. 
We use NCC as the basic match metric, thus the cost for matching pixel $p$ in $I_{1}$ with pixel $p+d$ in $I_{2}$ is

$$
C_{N C C}(p, d)=\frac{\sum_{q \in W_{p}}\left(I_{1}(q)-\bar{I}_{1}(p)\right) \cdot\left(I_{2}(q+d)-\bar{I}_{2}(p+d)\right)}{\sqrt{\sum_{q \in W_{p}}\left(I_{1}(q)-\bar{I}_{1}(p)\right)^{2} \cdot \sum_{q \in W_{p}}\left(I_{2}(q+d)-\bar{I}_{2}(p+d)\right)^{2}}},
$$

where $d=\left(d_{x}, 0\right)^{\prime}$ is the disparity, $W_{p}$ is the local aggregation window centered at pixel $p, \bar{I}_{k}(p)$ is the average intensity value of the pixels within the aggregation window centered at $p$. The effect of aggregation window size is twofold. The larger the aggregation window, the more robust the matching. But on the other hand, the aggregation window has to be small enough to avoid covering pixels from different depth levels. So we choose $5 \times 5$, which is moderate, to be the size of aggregation window throughout the paper. Equation (8) requires to compute the intensity differences between all the pixels in the local window and the average intensity for each (pixel, disparity) pair, which is too computationally expensive. So we use the fast NCC algorithm proposed by Lewis [14 in our system.

The NCC cost is then reversed and normalized into the range of $[0,1]$, with lower costs for more likely matches:

$$
\operatorname{Cost}(p, d)=\frac{1-C_{N C C}(p, d)}{2} .
$$

Since the images are taken from different view points, and are possibly with different sample quality, even each small local window has a unique intensity encoding in the original light pattern, error matches between $I_{1}$ and $I_{2}$ will still occur, especially where the surface curvature is large. So global stereo matching algorithms such as dynamic programming (DP) [15] have to be utilized for depth optimization. We find that DP is an effective approach to solve the problem of error matches caused by pixel sampling and noise.

The DP algorithm is processed along each scanline from left to right. For each (pixel, disparity) pair $(p, d)$, the accumulated cost is stored in the array $S$. Formally,

$$
\begin{aligned}
S(p, d)= & \operatorname{Cost}(p, d)+\min \left(S\left(p-(1,0)^{\prime}, d\right), S\left(p-(1,0)^{\prime}, d+(1,0)^{\prime}\right)+P_{1},\right. \\
& \left.\left.S\left(p-(1,0)^{\prime}, d+(-1,0)^{\prime}\right)+P_{1}, \min _{k} S\left(p-(1,0)^{\prime}, k\right)+P_{2}\right)\right),
\end{aligned}
$$

where cost $\operatorname{Cost}(p, d)$ is the NCC-based cost for matching pixel $p$ in $I_{1}$ to pixel $p+d$ in $I_{2}$. Discontinuities are not prohibited, but discouraged in our system. $P_{1}$ and $P_{2}$ are respectively the constant penalties for disparity discontinuity of 1 pixel and above 1 pixel. We empirically set $P_{1}$ to 0.2 and $P_{2}$ to 1.5 throughout our experiments.

After minimal total matching cost of the scanline is found, the disparity of each pixel $\mathrm{p}$ is taken as the one on the minimal cost path.

Since the disparities obtained from stereo matching are in pixel unit, the reconstructed shape will appear ladder-like if the disparities are directly used in 
triangulation. To solve this problem, for each pixel, we smooth its disparity value to sub-pixel accuracy by fitting a quadric surface within a $21 \times 21$ local window. Pixels that have far different disparity values with the center one are excluded in the fitting process. Outlier matches have less depth supports in their neighborhood, so they are easily detected and eliminated in the same process. The smoothed and outlier-free disparity map is then used as input in triangulation.

\section{Robust Least Square Triangulation}

Now we have corresponded any pixel $\mathbf{u}_{1}=\left(u_{1}, v_{1}, 1\right)^{\prime}$ in $I_{1}$ with a particular pixel $\mathbf{u}_{2}=\left(u_{2}, v_{2}, 1\right)^{\prime}$ in $I_{2}$. We can obtain the world coordinate $(X, Y, Z)^{\prime}$ corresponding to $\mathbf{u}_{1}$ by solving the following linear equations:

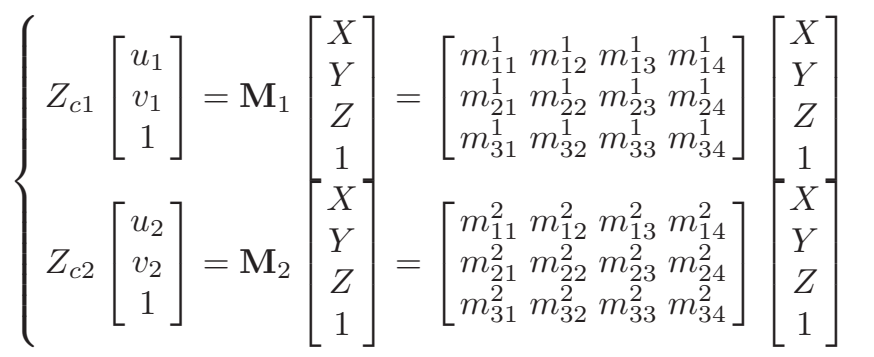

By eliminating $Z_{c 1}$ and $Z_{c 2}$, we will get four linear equations concerning about $X, Y$, and $Z$ :

$$
\left\{\begin{array}{c}
\left(u_{1} m_{31}^{1}-m_{11}^{1}\right) X+\left(u_{1} m_{32}^{1}-m_{12}^{1}\right) Y+\left(u_{1} m_{33}^{1}-m_{13}^{1}\right) Z=m_{14}^{1}-u_{1} m_{34}^{1} \\
\left(v_{1} m_{31}^{1}-m_{21}^{1}\right) X+\left(v_{1} m_{32}^{1}-m_{22}^{1}\right) Y+\left(v_{1} m_{33}^{1}-m_{23}^{1}\right) Z=m_{24}^{1}-v_{1} m_{34}^{1} \\
\left(u_{2} m_{31}^{2}-m_{11}^{2}\right) X+\left(u_{2} m_{32}^{2}-m_{12}^{2}\right) Y+\left(u_{2} m_{33}^{2}-m_{13}^{2}\right) Z=m_{14}^{2}-u_{2} m_{34}^{2} \\
\left(v_{2} m_{31}^{2}-m_{21}^{2}\right) X+\left(v_{2} m_{32}^{2}-m_{22}^{2}\right) Y+\left(v_{2} m_{33}^{2}-m_{23}^{2}\right) Z=m_{24}^{2}-v_{2} m_{34}^{2}
\end{array} .\right.
$$

This is an over-determined linear equation set. So $(X, Y, Z)$ is solved using least square error method [16], which is corresponding to finding the point with smallest sum of distances to the rays of two cameras.

\section{Experimental Results}

Our experimental setup consists of an NEC LT $245+$ projector working at $1024 \times$ 768 , two Olympus C-5060 cameras at $2592 \times 1944$, and a standard PC. Due to the depth blurring effect of the projector, although the resolution of our projector is $1024 \times 768$, we only use half its resolution in practice. A 2-D binary De Bruijn pattern with size $512 \times 384$ is scaled to fill the screen of projector, and thus every $2 \times 2$ square pixel unit represents a code bit. All the images taken by cameras are scaled to Video Graphics Array $($ VGA) size $(640 \times 480)$ for the processing followed. The algorithm is implmented in $\mathrm{C}++$ and run on a $\mathrm{PC}$ with $2.6 \mathrm{GHz}$ $\mathrm{CPU}$ and $1 \mathrm{~GB}$ memory. The depth acquisition process takes about $3 \sim 4$ seconds for each image pair. 


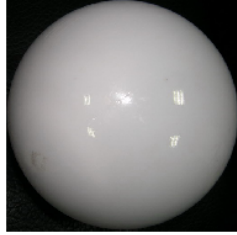

(a)

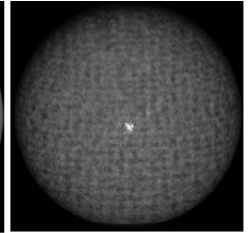

(b)

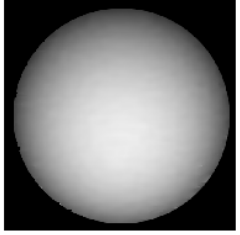

(c)

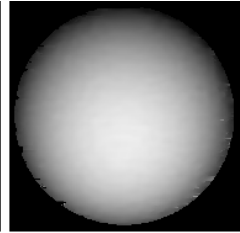

(d)

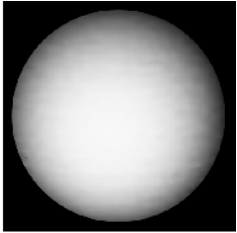

(e)

Fig. 5. (a) real object, (b) object illuminated by structured light pattern, (c, d) acquired depth maps and (e) reconstructed meshes of the experiment on a ball

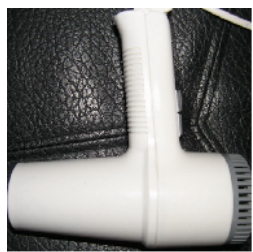

(a)

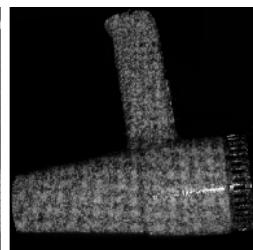

(b)

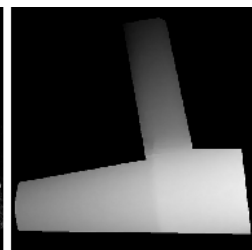

(c)

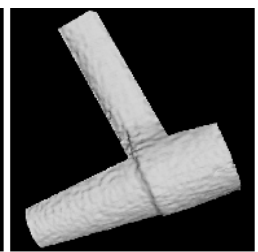

(d)

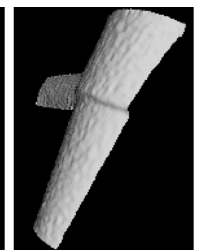

(e)

Fig. 6. (a) real object, (b) object illuminated by structured light pattern, (c) acquired depth maps and $(\mathrm{d}, \mathrm{e})$ two different views of the reconstructed meshes of the experiment on a hair drier

Firstly, the depth maps of a ball and a hair drier are reconstructed using our system. The real objects are shown in Fig. 5a and Fig.66, and the objects illuminated by the structured light are shown in Fig.5b and Fig.6b. The recovered depth maps using two calibrated cameras are shown in Fig. 56 and 6r. Fig. 6d shows the ball's depth map obtained by the alternative system using a calibrated projector and only one camera. There are more stereo matching errors near the object boundary, since in these regions, image $I_{p}$ and $I_{2}$ have much different pattern intensities and distortion. The reconstructed mesh of the ball is shown in Fig. 5e, and two different views of the reconstructed hair drier are shown in Fig. 6] and 66.

We find that the reconstructed meshes appear to be a little rough, especially where depth changes rapidly in Fig. 6 d and 6e. The main cause is that our stereo matching algorithm is based on pixel as searching unit. It is worthwhile to note that these results are obtained from only a single image pair.

In another experiment, a rotating cup is taken as the target object, which illustrates the system's ability to recover depth maps for moving objects. Due to space limitation, we only show two frames from the video sequence and their experimental results in Fig. 7. There are some characters in dark color (see Fig. 7b). Other structured light approaches base on pattern decoding such as 2-D binary grid [5] and colored De Bruijn sequence [7] will fail on such regions. On the contrary, our method gives robust reconstruction in such regions. The matching error to the right of the cup handle is caused by pattern occlusion in the other view. 


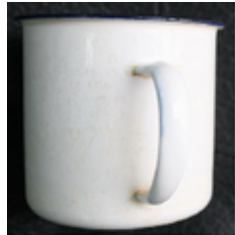

(a)

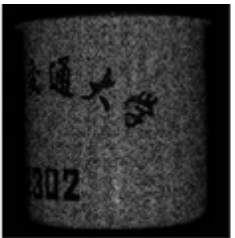

(b)

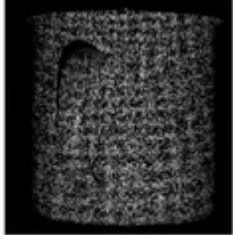

(c)

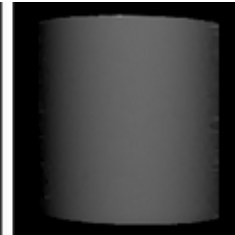

(d)

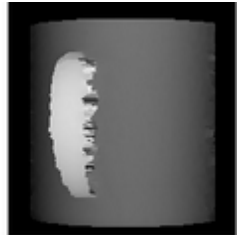

(e)

Fig. 7. (a) real object, (b, c) two frames of the object illuminated by structured light pattern and $(\mathrm{d}, \mathrm{e})$ acquired depth maps of the experiment on a rotating cup

\section{Conclusion and Discussions}

In this paper, we have proposed a new active stereo approach based on 2-D De Bruijn sequences. It has the advantage of handling the texture problem and acquiring depth maps of moving and deforming objects. 2-D De Bruijn sequences are emitted to the target object so that every small local window on the image is endowed with a unique code. But the difficulty of decoding these codes after camera's re-sampling is avoided by the use of NCC-based dense stereo matching. So there are few erroneous matches in the stereo matching stage.

Both the systems of using two calibrated cameras and one un-calibrated projector, and that using one calibrated camera and one calibrated projector are implemented. For geometrically calibrating the projector, we have proposed a novel reverse calibration method, which is validated by our experimental results. Good depth reconstruction results are obtained with both the systems.

A drawback of our system is the restriction to working only for indoor environments, due to the limitation of the energy emitted by the projector. This is a common limitation of active stereo.

In our current implementation, the processing speed is relatively slow for real time applications. By now, we can only record the moving objects with fixed cameras and reconstruct the depth maps offline. Hardware implementation of a real time online system is a challenging future work.

Acknowledgments. This work was supported in part by funds from China National Basic Research Program (973 Program 2006CB303105), Natural Science Foundation of China 60573147, and the collaboration research project with Omron Corporation, Japan.

\section{References}

1. Scharstein, D., Szeliski, R.: A Taxonomy and Evaluation of Dense Two-Frame Stereo Correspondence Algorithms. Int. J. Computer Vision 47(1-3), 7-42 (2002)

2. Brown, M.Z., Burschka, D., Hager, G.D.: Advances in Computational Stereo. IEEE Trans. Pattern Analysis and Machine Intelligence 25(8), 993-1008 (2003) 
3. Salvi, J., Pagès, J., Batlle, J.: Pattern Codification Strategies in Structured Light Systems. Pattern Recognition 37(4), 827-849 (2004)

4. Rusinkiewicz, S., Hall-Holt, O., Levoy, M.: Real-Time 3D Model Acquisition. In: SIGGRAPH 2002 Conference Proceedings, pp. 438-446 (2002)

5. Scharstein, D., Szeliski, R.: High-Accuracy Stereo Depth Maps Using Structured Light. In: IEEE computer society conference on computer vision and pattern recognition, vol. 1, pp. 195-202 (2003)

6. Lavoie, P., Ionescu, D., Petriu, E.M.: 3-D Object Model Recovery from 2-D Images Using Structured Light. IEEE Trans. Instrum. Meas. 53(2), 437-443 (2004)

7. Pagès, J., Salvi, J., Forest, J.: A New Optimised De Bruijn Coding Strategy for Structured Light Patterns. In: 17th Int. Conf. Pattern Recognition, vol. 4, pp. 284-287 (2004)

8. Morano, R.A., Ozturk, C., Conn, R., Dubin, S., Zietz, S., Nissanov, J.: Structured Light Using Pseudorandom Codes. IEEE Trans. Pattern Anal. Mach. Intell. 20(3), 322-327 (1998)

9. Tsai, R.Y.: A Versatile Camera Calibration Technique for High-Accuracy 3D Machine Vision Metrology Using Off-the-Shelf TV Cameras and Lenses. IEEE Trans. Robotics and Automation 3(4), 323-344

10. Zhang, Z.: A Flexible New Technique for Camera Calibration. IEEE Trans. Pattern Anal. Mach. Intell. 22(11), 1330-1334 (2000)

11. Heikkilä, J., Olli Silvén, O.: A Four-step Camera Calibration Procedure with Implicit Image Correction. In: IEEE Conf. Computer Vision and Pattern Recognition, pp. 1106-1112. IEEE Computer Society Press, Los Alamitos (1997)

12. Zeller, C., Faugeras, O.: Camera Self-calibration from Video Sequences: The Kruppa Equations Revisited. Research Report 2793, INRIA (February 1996)

13. Loop, C., Zhang, Z.: Computing Rectifying Homographies for Stereo Vision. In: Proc. IEEE Computer Science Conference on Computer Vision and Pattern Recognition, pp. 125-131 (1999)

14. Lewis, J.P.: Fast Normalized Cross-Correlation. In: Proceedings of Vision Interface (VI '95), pp. 120-123 (1995)

15. Cox, I.J., Hingorani, S.L., Rao, S.B., Maggs, B.M.: A Maximum Likelihood Stereo Algorithm. Computer Vision and Image Understanding 63, 542-567 (1996)

16. Press, W.H., Teukolsky, S.A., Vetterling, W.T., Flannery, B.P.: Numerical Recipes in C: The Art of Scientific Computing, 2nd edn., pp. 59-70. Cambridge University Press, Cambridge (1992) 